九州大学学術情報リポジトリ

Kyushu University Institutional Repository

\title{
Uber Die Einwirkung Von Hypohalogenit Auf Methylfurfural
}

Maekawa, Kazuyuki

Laboratory of Pesticide Chemistry, Faculty of Agriculture, Kyushu University

https://doi.org/10.5109/22619

出版情報 : 九州大学大学院農学研究院紀要. 9 (2)，pp.149-158，1949-05. Kyushu University バージョン：

権利関係 : 
Journal of the Faculty of Agriculture, Kyüshū Iniversity, Vol. 9 , No. 2 May 31, 1949

\title{
UBER DIE: EINWIRKUNG VON HYPOHALOGENIT AUF METHYLFURFURAL*
}

\author{
Kazuyuki Maekawa
}

Wenn Methylfurfural in einer 12\%igen Salzsàurelösung in kochendem Wasserbad erhitzt wird, so vermehrt sich die Menge der Substanz, die von der Natrium-hypojoditlösung reagiert wird, während sich die Menge des mit Phloroglucin niederfällbaren Methylfurfurals vermindert. Diese erstmals entdeckte Tatsache führte Verfasser vorläufig auf die Ringöffnung des Furankerns zurück, und kam dadurch zur fraktionierten jodometrischen Bestimmung von Furfural und Methylfurfural. ${ }^{(1)}$ So wird die leichtere Zersetzbarkeit von Methylfurfural als Furfural wohl dem Einflusse der $v$-ständigen Methylgruppe zukommen. Um weiteres darüber zu klären, untersuchte Verfasser hinsichtlich der Einwirkung von Hypohalogenit auf Methyl-furfural und -brenzschleimsäure.

Zur Entstehung des Haloforms aus Methylketon ist Enolisation von Keton in erster Linie unentbehrlich, und auf der folgenden Stufe setzt sich ihr strenges positives Wasserstoffatom mit Halo. genatom um. ${ }^{(2)(3)(4) /(f)(6)}$ Wénn man dieses nun zu Fällen von Methylfurfural und Methylbrenzschleimsäure anführt, so bedarf es der. Enolisation von der w-ständigen Methylgruppe für die Ent. stehung des Haloforms von beiden mit Hypo-bromit und -jodit. Für diese Enolisierbarkeit muss sich der Furankern entweder hydrolytisch ${ }^{(7)(g)}$ oder durch einen peroxydischen $Z$ wischenzustand $^{(9)(10)}$ oder in anderer Weise ${ }^{(11)(12)(13)}$ sprengen. Das Wasserstoffatom der $\omega$-ständigen Methylgruppe kann sich nämlich nicht an der Enolisation beteiligen, falls Sauerstoff in dem gebundenen;

\footnotetext{
* Vorgetragen vor dem Bezirksverein West-Japan von der Agr. Chem. Soc. Japan mit Prof. M. Hamada gemeinsam am 28. Feb. 1942; J. Agr. Chem. Soc. Japan, 18 (1942), 24 B. Untersuchungen über Furfural, IV. Mitteilung.
} 
brückenartigen Zustand gewesen ist. Wenn Methylfurfural sich in diesem Falle nach dem von Pummerer ${ }^{(s)}$ vorgeschlagenen Zersetungsprozess verhielte, so würde man Bẹrnsteinsäure gewinnen. In -Wirklichkeit isolierte Verfasser aber aus dem. Reaktionsgemisch von Hypohalit und Methylfurfural sowie Hypohalit und Methylbrenzschleimsäure Malcinsäure. Also wird es sich wohl in diesem Falle nicht hydrolytisch, sondern in anderer Weise, insbesondere unmittelbar Diketon bildend verhalten haben.

Als zweites Produkt von Methylfurfural und Methylbrenzschleimsaure wurde Bromoform sichergestelit und bestimmt. Die dabei gefundenen Werte ergeben, dass, falls $1 \mathrm{Mol}$. Methylfurfural und Methylbrenzschleimsäure $1 \mathrm{Mol}$. Bromoform liefert, $85 \%$ des Ausgangsmaterials umgewandelt wordẹn sind. (Während Jodoform mit Hypojodit nur 6\% ergeben hat.) Wenn die Reaktion von Methylfurfural und Hypobromit im Kaltgemische durchgeführt wird, so fin:det nur Oxydation von Methylfurfural zur Methyl. brenzschleimsäire statt, ohne weitere Reaktion.

Das im Reaktionsverlauf entwickelte Kohlensäuregas wurde auch sichergestellt und ruantitativ ermittelt aus folgenden Gründen; 1. als mittelbarer Hinweiss hinsichtlich der Entstehung von 1,4 Diketon, 2. um oxydative Zersetzung von $x$-Ketonsäure aufzuklären, und 3. um die. Tatsache ans Licht zu fördern, dass die w-ständige Methylgruppe der Decarboxylierungsfähigkeit der Karbonsäure teilhaft ist. Die dabei gefundenen' Werte ergeben. dass, falls $1 \mathrm{Mol}$. Probe $1 \mathrm{Mol} . \mathrm{CO}_{2}$ liefert, sich nur $30 \%$ von der theoretischen Ausbeute entwickeln.

Das verbrauchte Bromatom war auch hinsichtlich des Methylfurfurals und der. Methylbrenzschleimsäure bzw. etwa 7.5 Atome und 5.5 Atome. Dies zeigt, dass bei jenem sowohl Sprengung des Furankerns und Entstehung von Bromoform als auch Oxydation von Aldehyd zur Carboxylgruppe stattgefunden haben.

Zuletzt in Bezug auf frühere Mitteilung ${ }^{(1)}$ wurde die jodometrische Titration über die Methylbrenzschleimsäure sofort und nach einstündiger Erhitzung mit oder ohne verd. Salzsä̌ure ausgeführt. Die dabei gefundenen Ergebnisse zeigen, dass Methyl. brenzschleimsäure in wässriger Lösung eine sehr kleine Menge von Jod verbraucht hat, und also $2-4 \%$ davon in der mit Hypojodit oxydierbaren Form immer existieren. Wenn sie in wässriger Lösung erhitzt oder stehengelassen wird, so vermehrt sich freilich 
der Jodverbrauch. Erhitzt man auch Methylbrenzschleimsäure in $12 \%$ iger Salzsäurelösung, vermehrt sich der Iodverbrauch soweit und färbt sich so gelb bis orange ${ }^{(11\}}(\lambda=460 \mathrm{~m} / \ell)$. dass man damit beide, Brenzschleimsäure und Methylbrenzschleimsäure, unterscheiden kann, weil die erstere nicht verbraucht wird und sich nicht färbt.

Demnach folgert Verfasser hinsichtlich der Einwirkung von Hypohalit auf Methylfurfural und Methylbrenzschleimsäure folgendes;

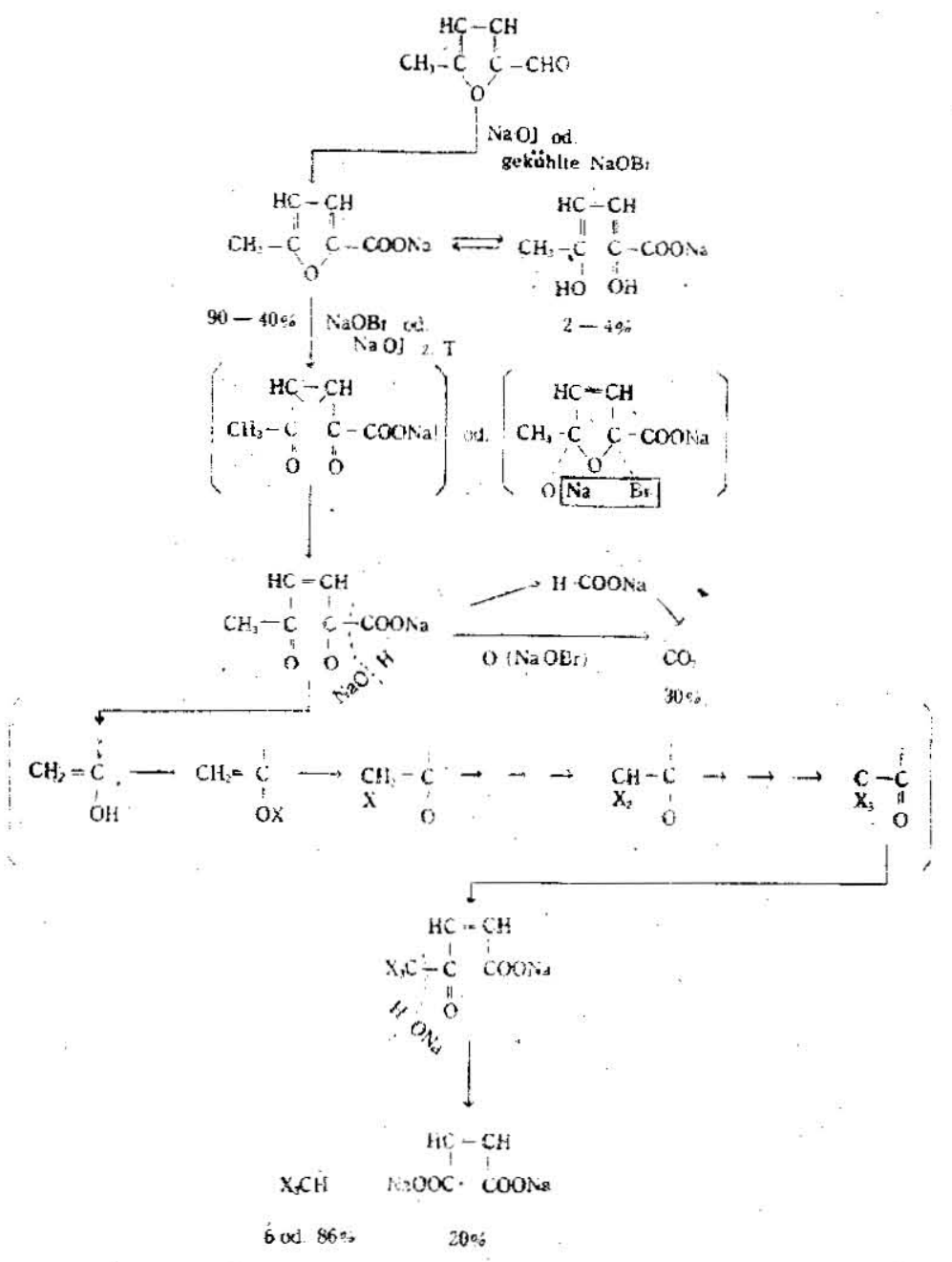


Me-furfural wird nämlich durch Hypojodit und abgekühltes Hypobromit zum Me-brenzschleimsäuressalz oxydiert, in welchem $2-4 \%$ in verdünnter wässriger Lösung (wie 10 milli $\mathrm{Mol}$.) als Form derjenigen 'vorhanden ist, wo mit Alkali und Jod Jodoform entstehen kann, und die Deformierung nach dieser Form beim Erhitzen mit 12\% iger Salzsäure gefördert wird. Hypobromit wirkt weiter auf Me-brenzschleimsäure ein; hierauf offnet sich der Furankern zuerst, und eine Seite der Spaltstücke, nämlich Methylketon wird sofort ${ }^{(6)|15\rangle}$ durch übriges Alkali enolisiert, schliesslich entsteht $\mathrm{CHBr}_{3}$; und die andere Seite entlässt unmittelbar oder nach dem Verlauf der Ameisensäure $\mathrm{CO}_{2}$ Gas. (Gleich falls entsteht $\mathrm{CO}_{2}$-Gas aus Ameisensäure $\left.{ }^{(16)}\right)$.

Verfasser folgert, dass auch bei Hypoiodit der Verlauf zum Teil in leicher Weise ist.

Die obengenannten Überlegungen könnten weiter für die Aufklärung gleicher Erscheinungen geltend gemacht werden, dass Pyronring, ${ }^{(17)}$ Laktonring ${ }^{(15)}$ usw. durch Hypohalit die Ringe ooffnen und dabei Haloform entsteht.

\section{BESCHREIBUNG DER VERSUCHF}

I. Einwirkung von Na-hypobromit auf Me-furfural und Me-brenzschleimsäure

(a) Zahl der verbrauchten Bromatome pro Mol. Subst.

Je $20 \mathrm{ccm}$ Bromlösung (enthielt $30 \mathrm{~g} \mathrm{KBr}$ und $8 \mathrm{~g} \mathrm{Br}_{2}$ im L, und wurde hinsichtlich ihrer gewissen Menge vorläufig $\mathrm{KJ}$ versetzt und mit $0.1 \mathrm{n}$. Thiosulfat titriert) und $0.5 \mathrm{n} \cdot \mathrm{NaOH}$ in veränder lieher Menge wurden in die Erlenmeyersche Flasche, die mit einem Stopfen versehen ist, gegossen, und die rote Farbe des Broms veränderte sich $\mathrm{zu}$ hellgelb. Dann wurden je $25 \mathrm{ccm} 1$ milli Mol. Lösung von Me-furfural bzw. Me-brenzschleimsäuresnatrium hinzugefügt und gut geschüttelt. Nach einstündigem Stehenlassen wird ein wenig Kaliumjodid zugesetzt, mit verd. Schwefelsäure angesäuert geschüttelt, und titriert dadurch frei werdendes Jod mit $0.1 \mathrm{n}$-Thiosulfat. Die dabei gefundenen Ergeb. nisse sind folgende ; 
Tabelle I. Einwirkung von $\mathrm{NaBrO}$ aut Me-furfural und Me-brenzschleimsäure.

\begin{tabular}{|c|c|c|c|c|c|c|}
\hline \multirow{3}{*}{$\begin{array}{c}0.5 \mathrm{n}-\mathrm{NaOH} \\
\text { hinzugefügt } \\
\text { in } \mathrm{ccm}\end{array}$} & \multicolumn{3}{|c|}{ Methylfurfural. } & \multicolumn{3}{|c|}{ Methylbrenzschleimsäure } \\
\hline & \multicolumn{3}{|c|}{ Bromverbrauch in } & \multicolumn{3}{|c|}{ Bromverbrauch in } \\
\hline & $\mathrm{c} \in \mathrm{m}$ & mg & Atome & $\mathrm{ccm}$ & $\mathrm{mg}$ & Atome \\
\hline $\begin{array}{l}4.00 \\
\text { (aquiv. zu Br) }\end{array}$ & 18.63 & 149.06 & 7.45 & 13.35 & 106.82 & 5.34 \\
\hline 4.25 & 18.63 & 149.06 & 7.45 & 13.50 & 108.03 & 5.40 \\
\hline 4.50 & 18.50 & 148.00 & 7.40 & 13.27 & 106.17 & 5.31 \\
\hline 5.00 & 18.35 & 146.80 & 7.34 & 13.63 & 109.04 & 5.45 \\
\hline 5.50 & 16.81 & 134.45 & $\ddot{6} .72$ & 13.15 & 10520 & 5.26 \\
\hline 6.00 & 12.38 & 99.05 & 4.95 & 13.39 & 107.18 & 5.38 \\
\hline 7.50 & 6.57 & 52.58 & 2.63 & 13.59 & 108.73 & 5.44 \\
\hline 10.00 & 5.46 & 43.67 & 2.18 & 18.23 & 145.82 & 7.29 \\
\hline
\end{tabular}

(10 com Br-lsg. $=80 \mathrm{mg} \mathrm{Br}_{2}$ )

Nämlich, durch Zusatz von theoretisch geforderter Alkalimenge verbraucht Mę-furfural etwa 7.5 Atome Brom und Me-brenzschleimsäuresnatrium nur etwa 5.5 Atome Brom. Aber durch weiteren Alkalizusatz ergibt sich ein umgekehrtes Verhältnis.

(b) Bestimmungen von entstandenem Bromoform.

$3.2 \mathrm{~g}$ Brom und $100 \mathrm{ccm} 0.5 \mathrm{n} \cdot \mathrm{NaOH}$ werden in birnförmigen Kolben mit einem unter ihrem Boden gestelten, in Grade eingeteilten "Rohr zugesetzt, dann wird $1.1 \mathrm{~g}$ Me-furfural (in $100 \mathrm{ccm}$ wässriger lösung) hinzugefügt und geschüttelt. Die klare Lösung wird bald trüb und scheidet zuletzt eine hellgelbe, schwere Lösung, nämlich $\mathrm{CHBr}_{3}, \mathrm{ab}$, das sich in dem unter dem Boden gestellten Rohr sammelt. Ausbeute etwa $0.75 \mathrm{ccm}$ (86\% der für $1 \mathrm{Mol}$. $\mathrm{CHBr}_{3}$ aus $1 \mathrm{Mol}$. Me-furfural berechneten Menge).

$$
\begin{aligned}
& \mathrm{K}_{\text {;00 }} 146.0^{\circ} \text { (korr.) } \mathrm{n}_{\mathrm{D}}^{18} 1.596 \quad \mathrm{D}_{1 \text { ) }}^{20} 2.8875 \\
& \text { 21.062,25.102 mg Sbst. : 46,871, } 55.824 \mathrm{mg} \mathrm{AgBr} \\
& \mathrm{CHBr}_{3} \text { Ber. Br. 94.86\% } \\
& \text { Gef. , } 94.70,94.64 \%
\end{aligned}
$$

(Anhang; Bestim. von der durch Elektolyse entstandenem Jodoform)

$1.1 \mathrm{~g}$ Me-furfural lost man in $100 \mathrm{ccm}$ Wasser, hierin wird $10 \mathrm{~g} \mathrm{KJ}$ und $5 \mathrm{~g} \mathrm{Na}_{2} \mathrm{CO}_{3}$ zugesetzt, und elektrolysiert(18);

Pt-Anod, $\mathrm{Pb}$-Kathod in der Tonzelle, Stromdichte-1 Ampère $/ \mathrm{Dm}^{2}$ und $\mathrm{CO}_{2}$-Gas einleitend. Elektrolysat wird in schwachem Alkali mit Ather ausgezogen und $\mathrm{CHJ}_{3}$ nach Slottaschem Verfahren(es) bestimmt.

Zwischen der Entstehung von $\mathrm{CHJ}_{3}$ und Alkalinitat des Reaktionsgemisches bestanden keine Beziehungen. Diese Ergebnisse zusammengefasst sind tolgende. 
(c) Zu $5.5 \mathrm{~g}$ Me-furfural (6.3 g Me-brenzschleimsäure) in 500 ccm Wasser gibt man bei Zimmertemperatur eine Lösung von $16 \mathrm{~g}$ Brom und $9.6 \mathrm{~g}$ Ätznatron in $200 \mathrm{ccm}$ Wasser. Nach eintäg. igem Stehen bei Zimmertemperatur wird die Lösung vom dem sich am Boden sammelnden Bromoform abgetrennt, einige Stücke $\mathrm{KJ}$ zugesetzt, mit verd. $\mathrm{H}_{2} \mathrm{SO}_{4}$ angesäuert, (wenn dabei Jod frei geworden ist, wird es mit Natriumsulfit reduziert) mit Natrium. sulfat gesättigt und etwa $20 \mathrm{mal}$ mit Äther ausgezogen. Der im Vacuum getrocknete Ätherrückstand erstarrt bald zu einem harten Kristall, der aus Wasser umkristailisiert wird, Ausbeute ca. $1 \mathrm{~g}$. F. 129.5, mit Maleinsäure keine Schmelzpunkterniederung zeigt.

$47.2^{\circ}, 86.5 \mathrm{mg} \quad$ Subst. verb. gegen Phenolpthalein ${ }^{1}$

$8.05,14.77 \mathrm{ccm} \quad \mathrm{n} / 10$ Natronlauge, während für Maleinsäure.

$8.14,14.91 \mathrm{ccm}$ berechnet wird.

Nachdem das aus Me-furfural und Bromlauge bestehende Reaktionsgemisch einige Minuten lang den Sonnenstrahlen ausgesetzt worden war, wurden Bariumchlorid, Ammoniak und Alkohol in einer 3 mal so grossen Menge zugesetzt. ${ }^{(12)(19)}$ Der dabei ausgefällte Niederschlag von Bariumsalz wurde filtriert, mit verd. Ammoniak gewaschen und wieder mit 5\%iger Salpeter säure aufgelöst. Die Säure wurde aus der von Halogen befreiten Flüssigkeit von $5 \%$ iger Salpetersäure mit Merkuronitrit in $5 \%$ iger Salpetersäure als Merkurofumarat gefält. Nach Stehenlassen im Eisschrank wurde der Niederschlag durch Glasfiltertiegel

Tabelle II. Jodoform aus Methylfurfural.

\begin{tabular}{|c|c|c|c|}
\hline \multicolumn{2}{|c|}{$\begin{array}{l}\text { Alkalinität der } \\
\text { Reaktionsgemische }\end{array}$} & $\operatorname{mg} \mathrm{CHJ}_{3}$ & Mol. $\mathrm{CHJ}_{3}$ \\
\hline $0.06 \mathrm{n}$ & $\left(\begin{array}{l}0.11 \mathrm{~g} \\
\text { Probe }\end{array}\right)$ & 21.8 & 0.06 \\
\hline $0.28 "$ & (" & 22.0 & $"=$ \\
\hline $0.53 "$ & (") & 21.9 & $"$ \\
\hline $1.80 "$ & i" ) & 22.2 & " \\
\hline \multirow{2}{*}{\multicolumn{2}{|c|}{$\begin{array}{l}\text { Bei Elektrolyse } \\
\text { (Probe 1.lg) }\end{array}$}} & 224.7 & " \\
\hline & & 221.1 & " \\
\hline
\end{tabular}

Alle zeigten nur $6 \%$ der für $1 \mathrm{Mol}: \mathrm{CHJ}_{3}$ aus $1 \mathrm{Mol}$. Me-furfural berechneten Menge. Wird Me-furfural in Salzsäurelösung erhitzt, so dabei wird die Zersetzung nach einer Richtung veranlassen, die Entstehung von $\mathrm{CHJ}_{3}$ mit Alkali und Jod vermehrt. 
abgesaugt, mit 5\% iger Salpetersänre, Wasser, Alkohol und Äther nacheinander saugend ausgewaschen und im Vak. über Schwefelsäure getrocknet. ${ }^{(20)}$

Für die Analyse ${ }^{(2)}$, wurde aus $15 \%$ iger Salpetersäure umkristallisiert und im Wasserofen getrocknet :

13.3, $81.4 \mathrm{mg}$ Subst. verb. $5.20,31.42 \mathrm{ccm}$ KCNS-Lösung, und diese entspr. $10.4,62.84 \mathrm{mg} \mathrm{Hg}$.

$$
\begin{array}{llll}
\text { Merkurofumarat } & \text { Ber. } & \mathrm{Hg} & 77.8 \% \\
& \text { Gef. } & , & 78.2,77.2 \%
\end{array}
$$

Wenn man vorläufig zu einer Bromlaugelösung, die in einem aus Kochsalz und Eis bestehendem Kaltgemische abgekühlt ist, die in gleicher Weise abgekühlte Me-furfurallösung gibt und über Nacht im-Eisschranke stehen lässt, só entsteht kein $\mathrm{CHBr}_{3}$. Dieses Reaktionsgemisch wird mit verd. $\mathrm{H}_{2} \mathrm{SO}_{4}$ angesäuert, und einigemal mit Äther extrahiert., Die Ätherlösurg wird verdampft, Alkohol zugegeben und Natriumbisulfit zugesetzt, um noch nicht reagiertes Me-furfural zu entfernen. Aus dem getrockneten Alkoholrückstand kamen alsbald Nadeln heraus, die aus Wasser umkristallisiert worden waren: Ausbeute $2.5 \mathrm{~g}$ aus $5.5 \mathrm{~g}$ Me-furfural. Mit Methylbrenzschleimsäure zeight es keine Schmełzpunkterniedrigung.

$79.4,95.3 \mathrm{mg}$. Subst. verb. gegen Phenolphthalein

$6.16,7.50 \mathrm{ccm}$ n/10-Natronlauge, während für

Me-brenzschleimsäure

$6.30 ., 7.56 \mathrm{ccm}$ berechnet werden.

(d) Bestimmung des Kohlensäuregas.

Das aus dem Reaktionsgemische abgespaltete Kohlensäuregas wurde in $\mathrm{n} / 10$-Barytalösung eingeleitet und über ihrem aliquoten Teil mit $0.1 \mathrm{n}$-Salzsäure gegen 'Phenolphthalein als Indikator titriert, ${ }^{(22)(23)}$ wobei die Korrektion mit $\mathrm{Na}_{2} \mathrm{CO}_{3}$ vorgenommen wurde. (24) So wurden je $10 \mathrm{ccm} 0.1 \mathrm{Mol}$. wässrige Lösungen von Me-furfural, Me-brenzschleimsäure, Furfural, Brenzschleimsäure und Ameisensäure in die Flasche hineingebracht, und es wurden $0.32 \mathrm{~g} \mathrm{Brom}$ und $0.2 \mathrm{~g}$ Ätznatron enthaltendes, $10 \mathrm{ccm}$ Wasser auf einmal hinzugefügt. Nach halbstündigem Stehen wurden KJ und verd. $\mathrm{H}_{2} \mathrm{SO}_{4}$ zugesetzt, und das entstandene Kohlensäuregas 
wurde in die Barytalösung durch Strömung der kohlensäurefreien Luft hineingetrieben. Die dabei gefundenen Werte sind folgende;

Tablle III, $\mathrm{CO}_{2}$-Abspaltung aus Me-furfural, Me-brenzschleimsäure Furfural, Brenzschleimsäure und Ameisensäure.

\begin{tabular}{lccc}
\multicolumn{1}{c}{$\begin{array}{c}\text { Substanz } \\
\mathrm{mg}\end{array}$} & $\begin{array}{c}\mathrm{n} / 10-\mathrm{Ba}(\mathrm{OH})_{2} \\
\text { verb. } \mathrm{ccm}\end{array}$ & $\begin{array}{c}\mathrm{CO}_{2} \\
\mathrm{mg}\end{array}$ & $\begin{array}{c}\mathrm{CO}_{0} \\
\% *\end{array}$ \\
Methylfurfural & 5.89 & 12.96 & 29.5 \\
Me-brenzschleirnsäure & 16.25 & 35.75 & 81.3 \\
Furfural $;$ & 7.12 & 15.66 & 35.6 \\
Brenzschleimsäure & 15.30 & 33.66 & 76.5 \\
Ameisensäure & 16.70 & 36.74 & 83.5
\end{tabular}

* Prozent der für $1 \mathrm{Mol}$. $\mathrm{CO}_{2}$ aus $1 \mathrm{Mol}$. Subst. berechneten Menge.

Dass es keinen Unterschied zwischen Furfural und Me-furfural in Bezug auf oxydative Decarboxylierung durch Natriumhypobromit gibt, zeigt, dass die Einwirkung der Bromlauge nach der Kern öffnung in ganz ähnlicher Weise verlaufen 'ist. Man könnte nämlich denken, dass die Konfigurationen, wie sie über der Decarboxylierung einen. Unterschied zeigen mögen, schon verloren waren-also die Furankerne'sie gleichfalls haben. Weil diè Ämeisensäure durch gleiche Behandiung $\mathrm{zu} \mathrm{CO} \mathrm{CO}_{2}$-Gas oxydiert wird, kann man noch nicht entscheiden, ob Ameisensäure aus einer Seite des den Kern geöffnetes Me-furfurals entsteht und dadurch $\mathrm{CO}_{2}$-Gas sich abspaltet.

\section{Tatsachen über die Öffnung des Furankerns bei Mé-brenzschleimsäure}

$\mathrm{Zu}$ je $50 \mathrm{ccm} 20$ milli Mol. wässrigèr Lösung von Me-brenz schleimsäure, welche aus Me-furfural durch Silberoxyd-Oxydation dargestellt und aus Wasser dreimal umkristallisiert wurde, werden n/50-Jodlösung und Alkali zugesetzt. Nach einstündigem Stehen oder Erhitżén auf dem Wasserbad unter Rückfluss wird mit verd. $\mathrm{H}_{2} \mathrm{SO}_{4}$ angesäuert und mit Thiosulfat zurücktitriert (I). Diese titrierten Lösungen werden zusammengegossen und in kongorotsaurer Lösung mit Äther ausgezogen. Die Ätherlösung wird abgedampft, und der ausgeschiedene Kristall wird aus Wasser umkristallisiert und getrocknet. Abermal wird diese Säure in 20 milli Mol. wässriger Lösung jodometrisch titriert (II). Diese titrier- 
ten Lösungen werden auch vereinigt und nach gleicher Weise noch einmal jodometrisch titriert (III).

Tabelle IV a. Jodverbrauch von Me-brenzschleimsàure.

Versuche
Nr.

(I)

(II)

(III)

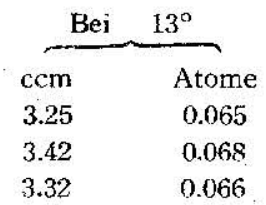

Tablle IV b.

Erhitzungsdauer
0
$30 \mathrm{Min}$
$60 \mathrm{"}$
$100 "$

\begin{tabular}{rr} 
ccm & Atome \\
3.28 & 0.066 \\
14.70 & 0.294 \\
31.25 & 0.625 \\
\multicolumn{2}{c}{ färbt sich rot }
\end{tabular}

Wie aus der obigen Tabelle erhellt, verbaucht Methylbrenz. schleimsäure bej Zimmertemperatur 0.06 Atome und nach Erhitzen 0.13 Atome Jod. Dies zeigt, dass der Stoff, der 3 Atome Jod verbraucht hat, zu 2-4\% gemischt ist, bzw. Methylbrenzschleimsäure in ihrer verd. wässrigen lösung immer $\mathrm{zu} 2-4 \%$ in dem Kern öffnendem Zustande, sozusagen in einem Gleichgewicht vorhanden ist.

Wenn Methylbrenzschleimsäure auch in 20 milli Mol. enthal tender, 12\%iger Salzsäurelösung unter Rückfluss auf dem kochenden Wasserbad während verschiedener Dauer erhitzt wird, danach je $50 \mathrm{ccm}$ der Lösung pipetiert und mit n/50-Jodiōsung und Ailkali in obengenannter Weise jodometrisch titriert wird,.so färbt sich diese, Methylbrenzschleimsäure enthaltende, Salzøäurelösung schon bei Erhitzung gelb bis orange (max. Absorp. $\lambda=460 \mathrm{~m} \mu$ ) und vermehrt den Jodverbrauch (Tabelle IV b), während die Brenzschleimsäure keine Erscheinungen zeigt.

Ich erlaube mir hiermit Herrn Professor M. Hamada für seine freundliche Anleitung und steten Ratschläge, die mir bei der Ausführung dieser und nachstehender Arbeit zuteil geworden sind, meinen herzlichsten Dank auszusprechen. Für die Möglichkeit zur weiteren Ausfübrung dieser Arbeit bin ich Herren Profesșoren Y. Oshima, K. Yamafuji und H. Iwata zu grossem Dank verpflichtet. Besten Dank schulde ich dem verstorbenen Herrn N. 
Fuiino, der mir unermüdliche Hilfe in dieser Untersuchung geleistet. hat. Dieser Versuch wurde mit Hilfei der Ausgaben des Unterrichtsministeriums für Wissenschaftliche Forschung durchgeführt. wofür der Verfasser an dieser Stelle Dank sagen möchte.

\section{LITERATUR}

(1) M. Hamada u. K. Maekawa, Protokoll d. japanischen Biochemischen Gesell schaft, 16 (1941), 1.

2) A. Pieroni u. E. Tonnioli. Gazz. Chem. Itai, 43 1. (1914), 620 [C. A. 8. (1914), 1273. ?

(3) F. Kröhnke, B., 69, (1936), 921.

(4) F. Straus u. R. Kühnel, B., 66, (1933), 1834.

(5) P. D. Bartlett, J. Am. Chem. Soc., 56, (1934), 967.

(6) J. Ssuknewitsch u. A. Tschilingarjan, C. A., 27. (1933), 2670: B., 68, (1935), $1210 ;$ B:, 69, (1936), 1537.

7) E. A. Kehrer u. P. Igler, B., 32, (1899), 1176.

(8) R. Pummerer, O. Gujot u. L. Birkofer, B., 68, (1935), 480.

(9) C. F. Cross, E. J. Bevan u. J. F. Briggș, B., 33, (1900), 3132.

(10) T. Yabuta u. R. Shimose, J. Agr. Chem. Soc. Japan, 4 (1928), 304

(11) N. A. Milas, J. Am. Chem. Soc., 49, (1927), 2005.

(12) N. A. Milas u. W. L. Walsh, ebenda, 57, (1935), 1389.

(13) F. Leger u. H. Hibbert. Can, J. Research, 16. 1938), (B). 68 [C. A., 32, 1938). 5788.]

(14) H. Sutter, Ann., 499, (1932), 55.

(15) K. Rehorst u. H. Scholz, B., 69, (1936), 520 ; IK. Rehorst, B, 71, (1938), 923

(16) E. Rupp, Archiv d. Pharmazie, 243, (1905), 69

(17) W. Feuerstein, B., 34, (1901), 1805.

(18) O. Dony-Hénault, Z. Elektrochem.. 7, (1900), 57

(19) N. A. Milas u. W. L. Walsh, J. Am. Chem. Soc., 61, (1939), 633

(20) A. Hahn u. W. Haarmann, Z. Biol., 87, (1927), 108.

(21) E. Stotz, J. Biol., 118, (1937), 471.

(22) B. Burkhart, L. Baur u. K. P. Link. J. Biol., 104, (1934), 171

(23) G. Lunde, E. Heen u. E. Öy, Kolloid Z., 83, (1938), 201.

(24) H. Friedman u. S. Morgulis, J. Am. Chem. Soc., 58, (1936). 911

(25) K. H. Slotta u. K. Neisser, Ber., 71 (1938), 1611. 